\section{Convection in a layered mantle}

\author{
from Peter J. Smith \\ Geomagnetism Correspondent
}

SEISMic data have revealed that the Earth's upper mantle contains several discontinuities in density and seismic velocity, the most prominent of which lies at a depth of about $650 \mathrm{~km}$. But what constraints does the existence of such discontinuities place on the form and magnitude of the convention currents which are believed to share the same region of upper mantle space? The full answer to this question would depend on a complete knowledge of the nature of the transitions producing the discontinuities - a matter which is far from being resolved. Insofar as the discontinuous changes in physical properties are due to mineralogical phase transitions they are relatively well understood; and their effect on convection is probably quite small. Richter (Rev. Geophys. Space Phys., 11, 223; 1973), for example, has shown that the Rayleigh-Benard convection driven solely by unstable vertical temperature gradients, the presence of the olivine-spinel phase transformation does not alter the structure of the flow and produces a roughly two-fold increase in steady amplitude. But if, in addition, chemical changes are involved, the problem changes because the position of the critical boundary will depend not on state parameters but on advection.

The question of whether or not the mantle is chemically inhomogeneous is still a matter of debate, although there must be a strong presumption in its favour. Press (in The Nature of the Solid Earth, McGraw-Hill, 1972), for example, investigated bulk sound velocity as a function of density for a set of Earth models obtained using Monte Carlo techniques and concluded that an offset in the velocity-density plot near the $650 \mathrm{~km}$ phase transition represents an increase in the $\mathrm{Fe} / \mathrm{Mg}$ ratio. Several other workers have reached similar conclusions, although such agreement is not necessarily as impressive as it seems because there are known to be certain problems associated with the determination of mean atomic weight using bulk velocity-density data. Moreover Wang (J. geophys. Res., 75, $3264 ; 1970$ ) has shown that it is possible to find an Earth model with a chemically homogeneous upper mantle which is quite consistent with free oscillation data. Anderson and Jordan (Phys. Earth Planet. Interiors, 3, 23; 1970 ), on the other hand, support iron enrichment of the lower mantle using several methods which are apparently not open to the objections directed at bulk velocity-density systematics.
In summary, then, chemical stratification in the upper mantle, though not proven, is highly likely; and so it is important to investigate what effects such layering is likely to have on convection processes. Richter and Johnson (J. geophys. Res., 79, 1635; 1974) have now attempted to do this by analysing the stability, to heating from below, of a system comprising two immiscible fluid layers of different density separated by a boundary which will be advected by the flow. What emerges from the fluid dynamical analysis is a stability diagram for the system showing the critical Rayleigh number (that is, the critical value of the normal Rayleigh number, $R_{a}$ ) for the most unstable mode as a function of $R_{p}$, the Rayleigh number based on the density contrast between layers. In other words, the stability diagram is a curve in $R_{11} R_{p}$ space which separates the stable region below (towards lower $R_{a}, R_{p}$ values) from the unstable region about. (towards higher $R_{u}, R_{\rho}$ values). In this context, stability means that at a given point in $R_{u}-R_{p}$ space all perturbations decay with time.

For $R_{\rho}$ lower than about $5 \times 10^{3}$, the most unstable mode is a standing oscillation on the interface between the two layers (overstable mode); and once the oscillatory motion begins, the fluid never returns to a state of total immobility. This overstable regime only exists within a very narrow band of $R_{a}$ values, however, suggesting that its occurrence is not very probable. Below this band (lower $R_{a}$ ) the system is stable, but above it ( $R_{a}$ higher than about $10^{*}$ ) direct convection throughout the entire depth of fluid (that is, throughout both layers) becomes possible. If such convection were actually to take place it would, of course, destroy the original chemical layering; conversely the persistence of a chemical boundary precludes total-depth convection. For $R_{\rho}$ higher than about $5 \times 10^{3}$ the situation is quite different. In this region the most unstable mode comprises separate convection within each layer and is thus independent of the density jump across the boundary between the layers. The stability curve separating the stable and unstable regions in $R_{u}-R_{\rho}$ space is now parallel to the $R_{f}$ axis, and so the stability or instability of the system depends only on the value of $R_{a}$.

These results place important bounds on acceptable Earth models. If the overstable mode is ruled out as improbable, for a model in which $R_{p}$ is lower than $5 \times 10^{3}$ the occurrence of convection is consistent with the continued presence of a chemical boundary because the convection would cross the boundary and chemically homogenise the system. Thus in a model in which both convection and chemical layering are inferred, $R_{p}$ must be higher than $5 \times 10^{3}$-and in this case there will be separate convection within each layer. In the case of the real Earth, much therefore depends on whether the $650 \mathrm{~km}$ discontinuity is just a phase change or whether it represents the combined effect of a phase transformation and a chemical change. Assuming that a chemical change is indeed present and that it involves an increase in iron relative to magnesium, several authors have calculated that $R_{\rho}$ would be of the order of $10^{\prime}$. This clearly implies that convention may only occur separately above and below a depth of about $650 \mathrm{~km}$.

This result does much to emphasise the importance of resolving the problem of whether or not the upper mantle is chemically inhomogeneous. The convective motions for seafloor spreading are likely to be those in the uppermost layer, and the thickness of this layer will influence the magnitude of the convective cells within it. Richter (Rev. Geophys. Space Phys., 11, 223; 1973), for example, showed that many of the possible flows in the mantle have horizontal scales comparable with the depth scale. The fact that the depth scale may be governed by the presence of chemical layering clearly makes it necessary to determine whether or not such layering actually exists.

\section{Modeling tide and surge interaction}

from J. Darbyshire

GREAT disasters and natural upheavals often provide an incentive for scientific research and this has been very true in the investigation of storm surges (or the extra tides caused by meteorological effects). The first such stimulus was probably the great Thames flood of 1928 which led to the systematic recording of these effects at Southend and to work on their prediction at the Bidston Observatory near Birkenhead. The methods originally used were largely empirical and the work of Doodson and Corkan was very important during this period.

The next great stimulus was the disastrous flooding of the English east coast and the Dutch coast during January 31-February 1, 1953, when many lives were lost and many of the Dutch dykes were seriously breached. After this there was set up at the British Meteorological Office, a flood warning system which made use of the fact that tidal abnormalities on the English side of the southern North Sea are usually preceded by similar smaller but detectable abnormalities at Aberdeen about $9 \mathrm{~h}$ earlier. This would give an even longer warning to the continental 